\title{
ČEBYŠEV SETS IN HYPERSPACES OVER A MINKOWSKI SPACE
}

\author{
Agnieszka Bogdewicz, Robert Dawson and Maria Moszyńska
}

Warsaw University of Technology, Poland, Saint Mary's University, Canada and Warsaw University, Poland

Dedicated to Professor Sibe Mardešić on the occasion of his 80th birthday

ABSTRACT. In this paper we extend our previous results on Čebyšev sets in hyperspaces over a Euclidean $n$-space to hyperspaces over a Minkowski space.

The notion of Čebyšev set has been studied mainly for normed linear spaces (see $[4,13]$ ), but it can be considered for arbitrary metric spaces (see [13, Appendix II]). A subset $A$ of a metric space $(X, \varrho)$ is a $\breve{C}$ ebyšev set in this space provided that for every point of $X$ there is a unique nearest point in $A$. The function $\xi_{A}: X \rightarrow A$ which assigns to $x \in X$ the unique nearest point of $A$ is called metric projection.

Čebyšev sets in $\mathcal{K}_{0}^{n}$ (the space of convex bodies in $\mathbb{R}^{n}$ ), $\mathcal{K}^{n}$ (the space of nonempty compact convex sets) and $\mathcal{O}^{n}$ (the space of compact, strictly convex sets), all endowed with the Hausdorff metric $\varrho_{H}$ associated with the Euclidean metric, were studied in $[2,5]$.

The present paper is closely related to [2] and [5]. Its purpose is to extend previous results on hyperspaces over a Minkowski space.

\section{Preliminaries}

Let $\|\cdot\|_{\circ}$ be the Euclidean norm in $\mathbb{R}^{n}$ :

$$
\|x\|_{\circ}:=\sqrt{x \circ x}
$$

where $\circ$ is the usual scalar product.

2000 Mathematics Subject Classification. 41A52, 52A20, 46 B99.

Key words and phrases. Convex body, strictly convex set, Čebyšev set, relative Čebyšev set, Minkowski space. 
Let $B^{n}$ and $S^{n-1}$ be the Euclidean unit ball and unit sphere:

$$
B^{n}:=\left\{x \in \mathbb{R}^{n} \mid\|x\|_{\circ} \leq 1\right\}, \text { and } S^{n-1}:=\left\{x \in \mathbb{R}^{n} \mid\|x\|_{\circ}=1\right\} .
$$

As usually, bd, cl, and int are boundary, closure, and interior, and $\operatorname{conv} A$ is the convex hull of $A$. For distinct $a, b$, let $\Delta(a, b)$ be the segment with endpoints $a, b$.

For any subset $A$ of $\mathbb{R}^{n}$, we shall use the symbol $[A]$ to denote the set of singletons in $A$ :

$$
[A]:=\{\{x\} \mid x \in A\} .
$$

Thus, in particular, $\left[\mathbb{R}^{n}\right]$ is the set of singletons in $\mathbb{R}^{n}$.

We shall use the symbol $\subset$ for strict inclusion:

$$
X \subset Y \Longleftrightarrow X \subseteq Y \text { and } X \neq Y \text {. }
$$

A Minkowski space is a finite dimensional Banach space $(M,\|\cdot\|)$ (see [14]). Thus, up to an isomorphism, every $n$-dimensional Minkowski space is a normed linear space $\left(\mathbb{R}^{n},\|\cdot\|\right)$.

Let $B$ be the unit ball determined by $\|\cdot\|$ :

$$
B:=\left\{x \in \mathbb{R}^{n} \mid\|x\| \leq 1\right\} .
$$

Then $B$ is a convex body symmetric at 0 . Conversely, every convex body $A$ symmetric at 0 determines a norm, $\|\cdot\|_{A}$, usually referred to as the Minkowski functional:

$$
\|x\|_{A}:=\inf \left\{t \in \mathbb{R}_{+} \mid x \in t A\right\}
$$

(see $\left[14\right.$, p. 17]). In particular, $\|\cdot\|_{B}=\|\cdot\|$ and the unit ball determined by $\|\cdot\|_{B}$ coincides with $B$.

Note that if the unit ball is strictly convex, then so are all the balls in $(M,\|\cdot\|)$.

We shall need the following lemma.

LEMMA 1.1. Let $x_{1}, x_{2} \in \mathbb{R}^{n}$ and $\alpha \geq \frac{1}{2}\left\|x_{1}-x_{2}\right\| ;$ let $\left(x_{1}+\alpha B\right) \cap\left(x_{2}+\alpha B\right)$ not be a singleton. If $\alpha_{0}$ is the radius of the smallest ball, $B_{0}$, with centre $x_{0}=\frac{1}{2}\left(x_{1}+x_{2}\right)$, containing $\left(x_{1}+\alpha B\right) \cap\left(x_{2}+\alpha B\right)$, then

(i) $\alpha_{0} \leq \alpha$;

(ii) $\alpha_{0}<\alpha$ if $\mathrm{bd} B$ does not contain any segment parallel to $x_{1}-x_{2}$.

Proof. Let $p \in\left(x_{1}+\alpha B\right) \cap\left(x_{2}+\alpha B\right) \cap \mathrm{bd}\left(x_{0}+\alpha_{0} B\right)$. Then there exist $b_{0} \in \operatorname{bd} B$ and distinct $b_{1}, b_{2} \in B$ such that

$$
p=x_{0}+\alpha_{0} b_{0}=x_{1}+\alpha b_{1}=x_{2}+\alpha b_{2} .
$$

Thus $\alpha\left(\frac{b_{1}+b_{2}}{2}\right)=\alpha_{0} b_{0}$, whence $\frac{\alpha_{0}}{\alpha}=\frac{\left\|b_{1}+b_{2}\right\|}{2} \leq 1$. This proves (i).

If $\operatorname{bd} B$ does not contain $\Delta\left(b_{1}, b_{2}\right)$, then the inequality is strict. This proves (ii). 
Let us first consider the family $\mathcal{C}^{n}$ of nonempty compact subsets of $\mathbb{R}^{n}$ and the family $\mathcal{C}_{0}^{n}$ of compact bodies (a member $A$ of $\mathcal{C}^{n}$ is a body whenever $A=\operatorname{cl} \operatorname{int} A$ ). Let $\varrho_{H}^{B}$ be the Hausdorff metric in $\mathcal{C}^{n}$ associated with the metric $\varrho^{B}$ induced by the norm $\|\cdot\|$ (compare $\left.[14]\right)$ :

$$
\varrho_{H}^{B}\left(A_{1}, A_{2}\right):=\max \left\{\overrightarrow{\varrho H}^{B}\left(A_{1}, A_{2}\right), \overrightarrow{\varrho H}^{B}\left(A_{2}, A_{1}\right)\right\},
$$

where the oriented Hausdorff metric $\vec{\varrho}_{H}^{B}$ is defined by the formula

$$
\overrightarrow{\varrho H}^{B}\left(A_{1}, A_{2}\right):=\inf \left\{\varepsilon>0 \mid A_{1} \subseteq A_{2}+\varepsilon B\right\}
$$

for every $A_{1}, A_{2} \in \mathcal{C}^{n}$.

Since

$$
\overrightarrow{\varrho H}^{B}\left(A_{1}, A_{2}\right)=\sup _{x \in A_{1}} \varrho^{B}\left(x, A_{2}\right),
$$

it follows that

$$
\varrho_{H}^{B}\left(A_{1}, A_{2}\right)=\max \left\{\sup _{x_{1} \in A_{1}} \varrho^{B}\left(x_{1}, A_{2}\right), \sup _{x_{2} \in A_{2}} \varrho^{B}\left(x_{2}, A_{1}\right)\right\} .
$$

Proof of (1.3) is the same as for the Euclidean case (see [8, 1.2.2]).

In what follows, $\mathcal{C}^{B}, \mathcal{C}_{0}^{B}, \mathcal{K}^{B}, \mathcal{K}_{0}^{B}$ and $\mathcal{O}^{B}$ are the families of nonempty compact subsets of $\mathbb{R}^{n}$, compact bodies, nonempty compact convex subsets, convex bodies, and strictly convex compact sets respectively, in each case endowed with $\varrho_{H}^{B}$.

\section{Invariant ČEBYŠev Sets in $\mathcal{K}_{0}^{B}$ And in $\mathcal{K}^{B}$}

Let us recall the notion of the minimal ring of a convex body (see $[1,7]$ ). Let $A \in \mathcal{K}_{0}^{n}$. For any $x \in A$, let $R_{A}(x)$ and $r_{A}(x)$ be, respectively, the radius of the smallest ball with centre $x$ containing $A$ and the radius of the biggest ball with centre $x$ contained in $A$. By a theorem of Bárány (proved much earlier by Bonnesen [3] for $n=2$ ), the function $f_{A}: A \rightarrow \mathbb{R}_{+}$defined by

$$
f_{A}(x):=R_{A}(x)-r_{A}(x)
$$

has a unique minimizer $x_{0}$, which belongs to $\operatorname{int} A$. This point $x_{0}$ is called the centre of the minimal ring of $A$; we shall denote it by $c(A)$.

Let

$$
R(A):=R_{A}(c(A)) \text { and } r(A):=r_{A}(c(A)) .
$$

Recall that for any two nonempty subsets $A_{0}, A_{1}$ of $\mathbb{R}^{n}$ the affine segment $\Delta\left(A_{0}, A_{1}\right)$ is defined by

$$
\Delta\left(A_{0}, A_{1}\right):=\left\{(1-t) A_{0}+t A_{1} \mid t \in[0,1]\right\}
$$

a family $\mathcal{X} \subset \mathcal{K}^{n}$ is affine convex provided that $\Delta\left(A_{0}, A_{1}\right) \subset \mathcal{X}$ whenever $A_{0}, A_{1} \in \mathcal{X}$.

According to [2, Theorem 2.2], 
The family $\mathcal{B}^{n}$ of Euclidean balls in $\mathbb{R}^{n}$ is an affine convex Čebyšev set in $\mathcal{K}_{0}^{n}$; for any $A \in \mathcal{K}_{0}^{n}$ the nearest ball has centre $c(A)$ and radius $\frac{1}{2}(R(A)+$ $r(A))$. The metric projection $\xi_{\mathcal{B}^{n}}$ is continuous.

The family $\mathcal{B}^{n}$ is invariant under the group Sim of similarities of $\mathbb{R}^{n}$.

Let now $\operatorname{Iso}_{B}$ be the group of isometries of $\left(\mathbb{R}^{n},\|\cdot\|_{B}\right)$. This group consists of all the affine transformations of $\mathbb{R}^{n}$ which map the unit ball $B$ onto its translate. ${ }^{1}$

Let, further, $\operatorname{Sim}_{B}$ be the group of similarities of $\left(\mathbb{R}^{n},\|\cdot\|_{B}\right)$ :

$$
h \in \operatorname{Sim}_{B} \Longleftrightarrow \exists f \in \operatorname{Iso}_{B} \exists t>0 \quad h=t f .
$$

For any subset $A$ of $\mathbb{R}^{n}$, let $\mathrm{I}_{B}(A)$ be the group of Minkowski selfisometries of $A$ :

$$
\mathrm{I}_{B}(A):=\left\{f \in \mathrm{Iso}_{B} \mid f(A)=A\right\} .
$$

We shall consider the family $\mathcal{B}$ of the Minkowski balls in $\left(\mathbb{R}^{n},\|\cdot\|_{B}\right)$ :

$$
\mathcal{B}:=\left\{x+t B \mid x \in \mathbb{R}^{n}, t>0\right\} .
$$

The following is evident.

Proposition 2.1. The family $\mathcal{B}$ is invariant under $\operatorname{Sim}_{B}$.

Let us note

Proposition 2.2. The family $\mathcal{B}$ is affine convex.

Proof. For every $t \in[0,1]$ and every two balls $B_{1}, B_{2}$ in $\left(\mathbb{R}^{n},\|\cdot\|_{B}\right)$, there exist $a_{1}, a_{2} \in \mathbb{R}^{n}$ and $\alpha_{1}, \alpha_{2}>0$ such that

$$
(1-t) B_{1}+t B_{2}=(1-t)\left(a_{1}+\alpha_{1} B\right)+t\left(a_{2}+\alpha_{2} B\right)=a+\alpha B,
$$

where $a=(1-t) a_{1}+t a_{2}$ and $\alpha=(1-t) \alpha_{1}+t \alpha_{2}$. Thus, the affine segment $\Delta\left(B_{1}, B_{2}\right)$ is a subset of $\mathcal{B}$.

We shall first consider hyperspace $\mathcal{K}_{0}^{B}$ of convex bodies, over a Minkowski space $\left(\mathbb{R}^{n},\|\cdot\|_{B}\right)$.

Carla Peri in [9] extended in the natural way the notion of minimal ring to arbitrary Minkowski spaces. ${ }^{2}$ In [10] among other results she obtained the following:

If the unit ball $B$ in a Minkowski space is strictly convex, then every convex body $A$ has a unique minimal ring with respect to $B$.

We refer to this unique minimal ring as $B$-minimal ring of $A$ and use the symbols $c^{B}(A), R^{B}(A)$, and $r^{B}(A)$ to denote centre, outer radius, and inner radius of the $B$-minimal ring of $A$.

\footnotetext{
${ }^{1}$ It may happen that the group $\operatorname{Iso}_{B} \cap G L(n)$ of linear Minkowski isometries consists of only two elements: identity and reflection at 0 (see [14, p. 14-17]).

${ }^{2}$ She uses the name "minimal shell" for minimal ring.
} 
The following theorem combined with Proposition 2.2 is a counterpart of [2, Theorem 2.2] (for continuity of metric projection, see Remark 4.4 and Proposition 4.5 below).

TheOREM 2.3. Let $\left(\mathbb{R}^{n},\|\cdot\|_{B}\right)$ be a Minkowski space with strictly convex unit ball B. Then

(i) the family $\mathcal{B}$ is an affine convex $\check{C}$ ebyšev set in $\mathcal{K}_{0}^{B}$. For every convex body $A$, the ball nearest to $A$ in the sense of $\varrho_{H}^{B}$ has centre $c^{B}(A)$ and radius $\frac{1}{2}\left(R^{B}(A)+r^{B}(A)\right)$,

(ii) for every convex body $A$,

$$
\varrho_{H}^{B}\left(A, \xi_{\mathcal{B}}(A)\right)=\frac{1}{2}\left(R^{B}(A)-r^{B}(A)\right) .
$$

Proof. The proof is analogous to that of [2, Theorem 2.2].

We are now looking for a counterpart of [2, Theorem 2.3], which states that every Čebyšev set in $\mathcal{K}_{0}^{n}$ invariant under Sim contains $\mathcal{B}^{n}$.

Notice that the proof of that theorem is based on the following characterization of Euclidean balls:

If a convex body $A$ in $\mathbb{R}^{n}$ is invariant under all linear isometries of $\mathbb{R}^{n}$, then $A$ is a ball with centre 0 .

Generally, a Minkowski ball $t B$ cannot be characterized as a convex body in $\mathbb{R}^{n}$ invariant under the linear Minkowski isometries. For instance, if $B$ is a cube, the group of linear isometries is a discrete group and there exist many centrally symmetric convex bodies invariant under these isometries. Thus a Minkowski space counterpart of [2, Theorem 2.3] must be proved differently.

For a Minkowski space $\left(\mathbb{R}^{n},\|\cdot\|_{B}\right)$, let

$$
\mathcal{C}_{B}:=\left\{C \in \mathcal{K}_{0}^{n} \mid \exists x \in \mathbb{R}^{n} \mathrm{I}_{B}(C)=\mathrm{I}_{B}(x+B)\right\} .
$$

So, $\mathcal{C}_{B}$ consists of all the convex bodies with the same group of Minkowski self-isometries as the balls.

TheOREM 2.4. Every Čebyšev set $\mathcal{X}$ in $\mathcal{K}_{0}^{B}$ invariant under $\operatorname{Sim}_{B}$ contains the orbit $\operatorname{Sim}_{B}(C)$ for some $C \in \mathcal{C}_{B}$.

Proof. It is easy to see that $\mathcal{C}_{B}$ is invariant under $\operatorname{Sim}_{B}$. Thus it suffices to prove that

$$
\mathcal{C}_{B} \cap \mathcal{X} \neq \emptyset \text {. }
$$

If $\mathrm{I}_{B}(A)=\mathrm{I}_{B}(x+B)$ for some $A \in \mathcal{X}$ and some $x \in \mathbb{R}^{n}$, then $A \in$ $\mathcal{C}_{B} \cap \mathcal{X} \neq \emptyset$. Assume that for every $A \in \mathcal{X}$ and every $x$

$$
\mathrm{I}_{B}(A) \subset \mathrm{I}_{B}(x+B),
$$

and suppose, to the contrary, that $\mathcal{C}_{B} \cap \mathcal{X}=\emptyset$. Take $C \in \mathcal{C}_{B}$ and let $A$ be the element of $\mathcal{X}$ nearest to $C$ with respect to $\varrho_{H}^{B}$. Then by (2.2), there exists $f \in \mathrm{I}_{B}(x+B)$ for some $x$ such that $f(A) \neq A$. 
Since $\mathrm{I}_{B}(x+B) \subseteq$ Iso $_{B}$ for every $x$, it follows that

$$
\varrho_{H}^{B}(C, A)=\varrho_{H}^{B}(f(C), f(A))=\varrho_{H}^{B}(C, f(A)),
$$

contrary to the assumption that $\mathcal{X}$ is a Cebyšev set in $\mathcal{K}_{0}^{B}$.

We shall now consider the hyperspace $\mathcal{K}^{B}$ of nonempty compact convex sets over a Minkowski space $\left(\mathbb{R}^{n},\|\cdot\|_{B}\right)$. For any nonempty compact convex set $A$, define $B$-Čebyšev centre of $A$ to be the centre $x$ of a minimal ball $x+\alpha B$ (i.e., a ball with minimal $B$-radius $\alpha$ ) containing $A .^{3}$ If $B$ is strictly convex, then such a point is unique $([6])$; we denote it by $\check{c}^{B}(A)$. Generally, the point $\check{c}^{B}(A)$ need not belong to $A$; as is well known, $\check{c}^{B}(A)$ belongs to $A$ for every $A \in \mathcal{K}^{n}$ if and only if either $n=2$ or $B=B^{n}$ (compare [6, p. 139]). We denote by $\check{R}^{B}(A)$ the $B$-radius of the minimal ball with centre $\check{c}^{B}(A)$ containing $A$.

The following theorem is a counterpart of [2, Theorem 3.3].

TheOREM 2.5. Let $\left(\mathbb{R}^{n},\|\cdot\|_{B}\right)$ be a Minkowski space with strictly convex unit ball B. Then

(i) $\left[\mathbb{R}^{n}\right]$ and $\mathcal{B} \cup\left[\mathbb{R}^{n}\right]$ are affine convex Č ebyšev sets in $\mathcal{K}^{B}$, invariant under $\operatorname{Sim}_{B}\left(\left(\mathbb{R}^{n},\|\cdot\|_{B}\right)\right)$; the metric projections are defined by the formulae

$$
\xi_{\left[\mathbb{R}^{n}\right]}(A):=\left\{\check{c}^{B}(A)\right\}
$$

and

$$
\xi_{\mathcal{B} \cup\left[\mathbb{R}^{n}\right]}(A):= \begin{cases}c^{B}(A)+\frac{1}{2}\left(r^{B}(A)+R^{B}(A)\right) B & \text { if } \operatorname{dim} A=n, \\ \check{c}^{B}(A)+\frac{1}{2} \check{R}^{B}(A) B & \text { if } 0<\operatorname{dim} A<n, \\ \{a\} & \text { if } A=\{a\}\end{cases}
$$

(ii) both metric projections are continuous.

Proof. The proof of (i) is analogous to those of [2, Theorems 3.2 and 3.3]. For (2.4) we apply Theorem 2.3 above.

(ii): Since for every two Minkowski spaces of the same dimension the associated Hausdorff metrics are uniformly topologically equivalent (see [14, p. 61]), it follows that for every Minkowski space $\left(\mathbb{R}^{n},\|\cdot\|_{B}\right)$ the space $\mathcal{K}^{B}$ is finitely compact, as it is for $\mathcal{K}^{n}$. Thus, metric projection on any Cebyšev set in $\mathcal{K}^{B}$ is continuous (compare [2, Proposition 1.6]).

As a counterpart of [2, Theorem 3.4] we obtain the following analogue of Theorem 2.4 above.

Theorem 2.6. Let $\mathcal{C}_{B}$ be defined by (2.1). Then every Čebyšev set $\mathcal{X}$ in $\mathcal{K}^{B}$ invariant under $\operatorname{Sim}_{B}$ contains $\left[\mathbb{R}^{n}\right] \cup \operatorname{Sim}_{B}(C)$ for some $C \in \mathcal{C}_{B}$.

\footnotetext{
${ }^{3} \mathrm{~A}$ Čebyšev centre is sometimes referred to as Čebyšev point; see $[2,8]$.
} 


\section{Families of translates in $\mathcal{K}^{B}$ and $\mathcal{O}^{B}$}

The family of singletons, $\left[\mathbb{R}^{n}\right]$, which is an example of Cebyšev set in $\mathcal{K}^{B}$ when the unit ball $B$ is strictly convex (see Theorem 2.5(i)), is the simplest example of a family of translates in $\mathcal{K}^{B}$. As was proved in [5] (see Proposition 3.5 and Remark 3.6), in the Euclidean case, this is the only possible example of a family $\left\{A+x \mid x \in \mathbb{R}^{n}\right\}$ which is a Cebyšev set in $\mathcal{K}^{n}$; if the set $A$ is not a singleton, the family of its translates is a Čebyšev set in $\mathcal{O}^{n}$ but generally not in $\mathcal{K}^{n}$.

The following theorem is a "Minkowski counterpart" of [2, Theorem 4.5], which concerns possible Čebyšev subsets of $\left[\mathbb{R}^{n}\right]$.

TheOREM 3.1. For a Minkowski space $\left(\mathbb{R}^{n},\|\cdot\|_{B}\right)$ with the unit ball $B$ the following are equivalent:

(i) the ball $B$ is strictly convex;

(ii) for every convex, closed subset $T$ of $\mathbb{R}^{n}$ with nonempty interior, the set $[T]$ of singletons is a Čebyšev set in $\mathcal{K}^{B}$;

(iii) there exists a convex, closed subset $T$ of $\mathbb{R}^{n}$ with nonempty interior such that $[T]$ is a Čebyšev set in $\mathcal{K}^{B}$.

Proof. (i) $\Longrightarrow$ (ii). We can follow the proof of [2, Theorem 4.5], because if $B$ is strictly convex, then in view of Lemma 1.1, for two balls $x_{1}+\alpha B$ and $x_{2}+\alpha B$ with nonempty intersection, the ball with centre $\frac{1}{2}\left(x_{1}+x_{2}\right)$, circumscribed over the intersection, has radius smaller than $\alpha$.

(ii) $\Longrightarrow$ (iii) is evident.

(iii) $\Longrightarrow($ i). Suppose, to the contrary, that $B$ is not strictly convex and let $T$ be as in (iii). Take an $x \in \operatorname{int} T$. There exists an $\alpha>0$ such that $B^{\prime}:=x+\alpha B \subseteq T$. Since $B^{\prime}$, as a homothet of $B$, is not strictly convex, its boundary contains a segment $\Delta\left(b_{1}, b_{2}\right)$. Since $x$ is the centre of $B^{\prime}$, also $\Delta\left(2 x-b_{1}, 2 x-b_{2}\right) \subseteq \mathrm{bd} B^{\prime}$. and let

Let $b_{0}:=\frac{1}{2}\left(b_{1}+b_{2}\right)$ and $v:=b_{1}-b_{0}$. Take a test set $X:=\Delta\left(b_{0}, 2 x-b_{0}\right)$

$$
x_{1}:=x+v \text { and } x_{2}:=x-v .
$$

It is easy to see that for every $t \in[0,1]$

$$
{\stackrel{\varrho_{H}}{ }}^{B}\left(\left\{(1-t) x_{1}+t x_{2}\right\}, X\right) \leq \alpha
$$

and

Thus

$$
\overrightarrow{\varrho H}^{B}\left(X,\left\{(1-t) x_{1}+t x_{2}\right\}\right)=\alpha .
$$

for every $t \in[0,1]$.

$$
\varrho_{H}^{B}\left(X,\left\{(1-t) x_{1}+t x_{2}\right\}\right)=\alpha
$$

On the other hand, for every $y \in T$

$$
\varrho_{H}^{B}(X,\{y\}) \geq \alpha,
$$


whence all the elements of $\Delta\left(\left\{x_{1}\right\},\left\{x_{2}\right\}\right)$ are nearest to $X$, i.e., [T] is not a Čebyšev set.

The following example shows that the assumption $\operatorname{int} T \neq \emptyset$ is essential for the implication (iii) $\Longrightarrow$ (i) above.

ExAmPle 3.2. Let $T:=\Delta(a,-a)$ for $a=\left(\frac{1}{2}, 0, \ldots, 0\right)$ and let $B:=B^{n} \cap$ $\left\{x=\left(x_{1}, \ldots, x_{n}\right) \mid x_{1} \in\left[-\frac{1}{2}, \frac{1}{2}\right]\right\}$. Take a test set $X \in \mathcal{K}^{n}$ and let

$$
\varrho_{H}(X,\{b\})=\varrho_{H}\left(X,\left\{b^{\prime}\right\}\right)=: \alpha>0
$$

for some $b, b^{\prime} \in T$. Then $X \subseteq(b+\alpha B) \cap\left(b^{\prime}+\alpha B\right)$, whence by Lemma 1.1 there exists $\alpha_{0}<\alpha$ such that $b_{0}+\alpha_{0} B \supset X$ for $b_{0}=\frac{1}{2}\left(b+b^{\prime}\right)$. Thus $\varrho_{H}\left(X,\left\{b_{0}\right\}\right) \leq \alpha_{0}<\alpha$. Hence $[T]$ is a Čbyšev set in $\mathcal{K}^{n}$, though $B$ is not strictly convex.

We now pass to families of translates in $\mathcal{O}^{n}$ (see [5]). We will need the following well known result:

Lemma 3.3. If $A_{1}, A_{2} \in \mathcal{O}^{n}$, then $A_{1}+A_{2} \in \mathcal{O}^{n}$.

THEOREM 3.4. For a Minkowski space $\left(\mathbb{R}^{n},\|\cdot\|_{B}\right)$ the following are equivalent:

(i) the ball $B$ is strictly convex;

(ii) for every $A \in \mathcal{O}^{n}$ the set $\mathcal{A}=\left\{A+x \mid x \in \mathbb{R}^{n}\right\}$ is a Čebyšev set in $\mathcal{O}^{B}$

(iii) there exists $A \in \mathcal{O}^{n}$ such that the set $\mathcal{A}=\left\{A+x \mid x \in \mathbb{R}^{n}\right\}$ is a Čebyšev set in $\mathcal{O}^{B}$.

Proof. The Euclidean version of the implication (i) $\Longrightarrow$ (ii) coincides with [5, Theorem 3.3]. The only property of the ball $B^{n}$ used in the proof of that theorem is strict convexity of $A+\alpha B^{n}$ for every $A$ strictly convex ([5, Proposition 1.3]). In view of Lemma 3.3, the Minkowski sum of two strictly convex sets is strictly convex. Thus (i) $\Longrightarrow$ (ii).

(ii) $\Longrightarrow$ (iii) is evident.

(iii) $\Longrightarrow$ (i). Suppose, to the contrary, that (iii) holds and $B$ is not strictly convex. In view of the implication (iii) $\Longrightarrow$ (i) in Theorem 3.1 we may assume that $A$ is not a singleton.

Let $\Delta\left(b, b^{\prime}\right) \subset \operatorname{bd} B$ and so $\Delta\left(-b^{\prime},-b\right) \subset \operatorname{bd} B$. Let $b_{1}=\frac{1}{2}\left(b+b^{\prime}\right), b_{2}=$ $-b_{1}$, and $u=\frac{b-b^{\prime}}{\left\|b-b^{\prime}\right\|}$.

We shall construct a strictly convex body $C \subset B$ such that

(a) $C$ is not contained in any ball $t B$ for $t<1$,

(b) there exists $t_{0}>0$ such that $0 \in C+t u \subseteq B$ for every $t \leq t_{0}$.

Let $B_{0}$ be the Euclidean ball with centre 0 and radius $r=\frac{1}{4}\left\|b-b^{\prime}\right\|$ and let $H$ be a linear hyperplane orthogonal to $b_{1}-b_{2}$. For every $c \in H \cap \operatorname{bd} B_{0}$ 
there exists a unique circle passing through $b_{1}, b_{2}, c$. Let $L_{c}$ be the arc of this circle with endpoints $b_{1}, b_{2}$. We define

$$
C:=\operatorname{conv} \bigcup\left\{L_{c} \mid c \in H \cap \operatorname{bd} B_{0}\right\} .
$$

It is easy to check that $\mathrm{bd} C \backslash\left\{b_{1}, b_{2}\right\}$ consists of elliptic points (i.e., points with positive Gauss curvature), whence $C$ is strictly convex. Evidently conditions (a) and (b) are satisfied.

Let now $X:=A+C$. This test body is strictly convex because both $A$ and $C$ are. To prove that there is more than one translate of $A$ nearest to $X$, it suffices to show that there is more than one translate of $X$ nearest to $A$.

Let $t_{0}$ be as in (b). Since

$$
\vec{\varrho}_{H}^{B}(X+t u, A)=\vec{\varrho}_{H}^{B}(C+t u,\{0\})=\inf \{\alpha>0 \mid C+t u \subseteq \alpha B\},
$$

by (a) and (b) it follows that

$$
\vec{\varrho}_{H}^{B}(X+t u, A)=1
$$

for all $t \leq t_{0}$.

On the other hand, by (b), the origin belongs to $C+t u$ for sufficiently small $t$, whence there exists $t_{1}>0$ such that for $t \leq t_{1}$

$$
\vec{\varrho}_{H}^{B}(A, X+t u)=\vec{\varrho}_{H}^{B}(\{0\}, C+t u)=0 .
$$

Hence, for all $t \leq \min \left\{t_{0}, t_{1}\right\}$,

$$
\varrho_{H}^{B}(X+t u, A)=1,
$$

a contradiction.

\section{FinAl REMARKS AND OPEN PROBLEMS}

REMARK 4.1. One of the main results of [5] concerns strictly nested families in $\mathcal{C}^{n}([5$, Theorem 2.5]). Let us observe that no Euclidean property of the unit ball $B^{n}$ was used in [5, Section 2]; hence the statements $2.5-2.9$ in [5] remain valid in arbitrary Minkowski space with a unit ball $B$. In particular,

- Every closed, dense, strongly nested family in $\mathcal{C}^{B}$ is a Čebyšev set relative to $\mathcal{K}^{B}$.

- No nested family is a Čebyšev set in $\mathcal{C}^{B}$ or in $\mathcal{C}_{0}^{B}$.

REMARK 4.2. Theorem 5.2 in [2] is valid for arbitrary Minkowski space: Every strictly affine convex subfamily of $\mathcal{K}^{n}$ is a Čebyšev set in $\mathcal{K}^{B}$.

Remark 4.3. Proposition 4.7 in [2] can be extended over Minkowski spaces with strictly convex unit ball:

If $B$ is strictly convex, then no ball in $\mathcal{K}^{B}$ is a Čebyšev set. 
REMARK 4.4. Theorem 2.2 in [2] contains information about continuity of the metric projection, while Theorem 2.3 above does not. The reason is that the argument used in proof of [2, Theorem 2.2] is based on some special properties of the Euclidean space. However, the continuity of $\xi_{\mathcal{B}}$ can be easily deduced from the continuity of the metric projection of $\mathcal{K}^{B}$ onto the closure of $\mathcal{B}$ (see Theorem 2.5 above).

Proposition 4.5. If $\left(\mathbb{R}^{n},\|\cdot\|_{B}\right)$ has strictly convex unit ball $B$, then the metric projection $\xi_{\mathcal{B}}$ is continuous.

Proof. Evidently,

$$
\xi_{\mathcal{B}}=\xi_{\mathcal{B} \cup\left[\mathbb{R}^{n}\right]} \mid \mathcal{K}_{0}^{B}
$$

Thus the assertion follows directly from Theorem 2.5(ii).

PROBLEM 4.1. Is strict convexity of B necessary for existence of Čebyšev sets in $\mathcal{K}_{0}^{B}$ and $\mathcal{K}^{B}$ invariant under $\operatorname{Sim}_{B}$ ?

\section{REFERENCES}

[1] I. Bárány, On the minimal ring containing the boundary of a convex body, Acta Sci. Math. (Szeged) 52 (1988), 93-100.

[2] A. Bogdewicz and M. Moszyńska, Čebyšev sets in the space of convex bodies, Rend. Circ. Mat. Palermo (2), Suppl. 77 (2006), 19-39.

[3] T. Bonnesen, Über das isoperimetrische Defizit ebener Figuren, Math. Ann. 91 (1924), 252-268.

[4] D. Braess, Nonlinear Approximation Theory, Springer-Verlag, Berlin, 1986.

[5] R. J. MacG. Dawson and M. Moszyńska, Čebyšev sets in hyperspaces over $\mathbb{R}^{n}$, Canad. J. Math., to appear.

[6] A. L. Garkavi, On the Čebyšev centre and convex hull of a set, Uspekhi Mat. Nauk 19(6) (1964), 139-145, in Russian.

[7] M. Moszyńska, Remarks on the minimal rings of convex bodies, Studia Sci. Math. Hungar. 35 (1999), 155-174.

[8] M. Moszyńska, Selected topics in convex geometry, Birkhäuser Boston, Inc., Boston, 2006.

[9] C. Peri, On the minimal convex shell of a convex body, Canad. Math. Bull. 36 (1993), 446-472.

[10] C. Peri, Minimal shells containing a convex surface in Minkowski space, Manuscripta Math. 90 (1996), 333-342.

[11] R. Schneider, Pairs of convex bodies with unique joining metric segment, Bull. Soc. Roy. Sci. Liège 50 (1981), 5-7.

[12] R. Schneider, Convex Bodies: the Brunn-Minkowski Theory, Cambridge University Press, Cambridge, 1993.

[13] I. Singer, Best Approximation in Normed Linear Spaces by Elements of Linear Subspaces, Springer-Verlag, New York-Berlin, 1970.

[14] A. C. Thompson, Minkowski Geometry, Cambridge University Press, Cambridge, 1996. 
A. Bogdewicz

Department of Mathematics

Warsaw University of Technology

Pl. Politechniki 1, 00-661 Warszawa

Poland

E-mail: abogde@mini.pw.edu.pl

R. J. MacG. Dawson

Department of Mathematics and Computing Science

Saint Mary's University

Halifax, Nova Scotia

Canada

E-mail: rdawson@cs.stmarys.ca

M. Moszyńska

Institute of Mathematics

Warsaw University

ul. Banacha 2, 02-097 Warszawa

Poland

E-mail: mariamos@mimuw.edu.pl

Received: 11.9.2006.

Revised: 8.2.2007. 